\title{
Further evidence for the genetic association between CACNA1I and schizophrenia
}

\author{
Yijun Xie ${ }^{1 \dagger}$, Di Huang ${ }^{2 \dagger}$, Li Wei $^{3 \dagger}$ and Xiong-Jian Luo ${ }^{1,2^{*}}$
}

\begin{abstract}
Background: Recent large-scale genome-wide association studies (GWAS) have showed that the neuronal calcium signaling has pivotal roles in schizophrenia (SCZ) in populations of European of ancestry. However, it is not known if calcium signaling pathway genes are also associated with SCZ in Han Chinese population.

Methods: Here we investigated the association between genetic variants in three calcium signaling pathway genes (CACNB2, CACNA1C and CACNA1I) and SCZ in 1615 SCZ cases and 1597 controls.

Results: A single nucleotide polymorphism (SNP) (rs4522708) in CACNA1/ is significantly associated with SCZ in our Chinese sample $\left(O R_{A}\right.$ allele $=1.19$, corrected $\left.P=0.042\right)$, suggesting that CACNA1/ may also be a risk gene for SCZ in Chinese population. Of note, the risk allele (A allele) of SNP rs4522708 is same in European and Chinese populations. Meta-analysis of Chinese and European samples further strengthened the association of rs4522708 with SCZ $\left(\mathrm{OR}_{\mathrm{A}}\right.$ allele $\left.=1.074, P=6.26 \times 10^{-11}\right)$. Expression analysis showed that CACNA1/ was significantly up-regulated in hippocampus of SCZ cases compared with controls, implying that dysregulation of CACNA1/ may have a role in schizophrenia pathogenesis.
\end{abstract}

Conclusions: Our study suggests that CACNA1l is a risk gene for SCZ in Chinese population and provides further evidence that supports the potential role of neuronal calcium signaling in schizophrenia.

Keywords: Schizophrenia, CACNA1l, Genetic association, Gene expression

\section{Background}

Schizophrenia (SCZ) is a severe mental disorder that affects about $1 \%$ of the world's population [1]. The core symptoms of SCZ include delusions, hallucinations and cognitive dysfunction [2,3]. Adoption, family and twin studies indicated that SCZ has a strong genetic component [4]. The heritability of SCZ was estimated about $80 \%[4,5]$, suggesting that genetic factor plays a crucial role in SCZ. Though numerous genetic linkage and association studies have been carried out in different ethnic populations and multiple susceptibility genes have been

\footnotetext{
*Correspondence: luoxiongjian@mail.kiz.ac.cn

${ }^{\dagger}$ Equal contributors

${ }^{1}$ Clinical Laboratory, Sichuan Academy of medical sciences \& Sichuan provincial people's hospital, Chengdu 610072, China

${ }^{2}$ Key Laboratory of Animal Models and Human Disease Mechanisms of the Chinese Academy of Sciences \& Yunnan Province, Kunming Institute of Zoology, Chinese Academy of Sciences, Kunming, Yunnan 650223, China Full list of author information is available at the end of the article
}

identified [6-8], only limited risk genes have been consistently replicated in diverse populations. The advent of genome-wide association studies (GWAS) provides an opportunity to dissect the genetic basis of SCZ. In the past decade, multiple GWAS have been performed in world's populations and multiple promising risk variants and genes have been reported [9-13]. In 2014, the schizophrenia working group of the psychiatric genomics consortium (PGC2) conducted a large-scale meta-analysis of GWAS through using 36,989 SCZ cases and 113,075 controls [13]. Over $100 \mathrm{SCZ}$-associated loci have been identified by PGC. A detailed characterization of the risk loci showed that calcium signaling pathway genes may play pivotal roles in SCZ [13]. Two calcium signaling pathway genes (including CACNA1C and CACNA1I) showed robust association with $\mathrm{SCZ}$ in PGC2, strongly implying these genes may be involved in $\mathrm{SCZ}$ pathogenesis. In addition, another calcium signaling pathway gene 
(CACNB2) was also reported to be associated with $\mathrm{SCZ}$ [14]. A recent trans-ancestry meta-analyses of Chinese and PGC2 samples (a total of 43,175 cases and 65,166 controls) further supports the association between CACNB2, CACNA1C, CACNA1I genes and schizophrenia [15]. These findings strongly suggest that neuronal calcium signaling pathway is involved in schizophrenia. To further investigate if these calcium signaling pathway genes are also associated with SCZ in Han Chinese population, we performed a replication study through recruiting 1615 SCZ cases and 1597 controls. We first genotyped three SNPs (one SNP in CACNB2, one SNP in CACNA1C and one SNP in CACNA1I) and tested their associations with $\mathrm{SCZ}$ in a Chinese sample. We then explored the expression level of these three genes in schizophrenia cases and controls. Our study indicates that a SNP in CACNA1I is significantly associated with $\mathrm{SCZ}$ in Chinese population, suggesting that CACNA1I is also a risk gene for SCZ in Han Chinese.

\section{Materials and methods Study subjects}

We included 1615 SCZ cases and 1597 controls in this study. All of SCZ cases were from local mental health hospitals (or centers) and some of the patients have been reported in our previously studies [16, 17]. Diagnosis was finished by at least two experienced psychiatrists with DSM IV criteria. $61.3 \%$ of the SCZ cases were females and the ages of the SCZ cases range from 13 to 81 years (35.1 \pm 13.26 years). Patients with history of head injuries and drug abuse were excluded in this study. Healthy controls were local residents and screened for lifetime history of mental illness. $63.9 \%$ of the controls were females and the ages of the controls range from 17 to 74 years $(38.08 \pm 12.31$ years). Written informed consents were obtained from all the studied subjects and this the research was approved by the internal review board of Kunming Institute of Zoology, CAS. More detailed information about the sample description can be found in our previous studies [16, 17].

\section{SNP selection}

As the goal of this study is to explore if the calcium signaling pathway genes reported in European populations are also associated with SCZ in Han Chinese, we selected three previously reported risk genes for SCZ, including CACNB2 [14], CACNA1C and CACNA1I [13]. All of these three genes encode voltage-dependent calcium channel subunits. CACNB2 encodes voltagedependent L-type calcium channel subunit beta-2. CACNA1C encodes $\mathrm{Ca}_{\mathrm{v}} 1.2$, an alpha- 1 subunit of a voltage-dependent calcium channel. And CACNA1I encodes $\mathrm{Ca}_{\mathrm{v}} 3.3$, an alpha-1I subunit of a voltage-dependent calcium channel. For CACNB2, we selected SNP rs4748478, which showed significant association with SCZ in PGC2 $\left(P=8.0 \times 10^{-3}\right)$. For $C A C N A 1 C$, we selected SNP rs1006737, which frequently reported to be associated with SCZ in previously studies [18-22]. For CACNA1I, we selected SNP rs4522708, which is also significantly associated with SCZ in PGC2. Of note, rs1006737 and rs4522708 reached genome-wide significance level in PGC2 $\left(P=1.09 \times 10^{-16}\right.$ and $P=2.41 \times 10^{-16}$, respectively) [13].

\section{Genotyping}

SNP genotyping was performed using SNaPShot method as described previously [16]. Single nucleotide extension strategy was used for genotyping. Briefly, DNA fragments containing the SNP site were amplified first using PCR. The PCR products were then treated with Shrimp Alkaline Phosphatase (SAP) and Exonuclease I (ExoI), which remove primers and unincorporated dNTPs. The cleaned PCR products were then used as templates for single nucleotide extension. The genotyping primers were designed to stop just one base upstream of the interest SNP. As ddNTPs were used, so the polymerase extends the primer by one nucleotide, adding a single ddNTP to its $3^{\prime}$ end. ABI 3730 was used to determine which base was added. More detailed information about SNaPShot genotyping method can be found in our previous study [16].

\section{Statistical analysis}

We used PLINK (v1.9) [23] to perform the association test (chi-square allelic test with $1 \mathrm{df}$ ). Bonferroni correction was used to correct the $P$ values. Meta-analysis was performed using metafor package (http://www.metaforproject.org/) [24] implemented in $\mathrm{R}$ as described previously [25] and Fixed-effect model was used.

\section{Expression analysis of CACNA1I in SCZ cases and controls} To explore if expression of these CACNA1I was changed in SCZ cases compared with controls, we examined the expression level of CACNA1I in SCZ subjects and controls using GES53987 [26]. In brief, 19 SCZ cases and 19 controls were included in GSE53987 and gene expression in hippocampus was measured. The raw expression values were processed as described in the original study [26] and student $t$ test was used to determine if expression of CACNA1I is significantly changed in SCZ cases compared with controls.

\section{Linkage disequilibrium analysis}

Linkage disequilibrium (LD) analysis were performed using genotype information from the 1000 genomes project [27]. As all of the subjects included in this study were Han Chinese, we only used the genotype information of 103 Chinese subjects (CHB) from the 1000 genomes project to construct and calculate LD values ( $r$ [2]) among the SNPs. Haploview program was used to calculated LD [28]. 
Table 1 Association significance between the studied SNPs and schizophrenia

\begin{tabular}{|c|c|c|c|c|c|c|c|c|c|}
\hline SNP id & Chr & $\mathrm{A} 1^{\mathrm{a}}$ & $A 2^{b}$ & Freq_A $A^{c}$ & Freq_U $U^{d}$ & $x^{2 e}$ & $\mathrm{OR}^{9}$ & $P$ & $P_{\text {corrected }}^{\mathrm{f}}$ \\
\hline rs4748478 & 10 & G & A & $49.5 \%$ & $48.8 \%$ & 0.299 & 1.028 & 0.585 & 1.0 \\
\hline rs1006737 & 12 & A & G & $5.43 \%$ & $4.96 \%$ & 0.725 & 1.102 & 0.395 & 1.0 \\
\hline rs4522708 & 22 & A & G & $86.11 \%$ & $83.92 \%$ & 5.981 & 1.188 & 0.0145 & 0.0435 \\
\hline
\end{tabular}

${ }^{a}$ Reference allele. ${ }^{b}$ Alternative allele. ${ }^{c}$ Frequency of reference allele in cases, ${ }^{d}$ Frequency of reference allele in controls. ${ }^{e} \mathrm{Chi}$ square value. ${ }^{\mathrm{f}} \mathrm{P}$ was corrected by Bonferroni correction. ${ }^{9}$ Odds ratio is based on reference allele. Significant associations were shown in bold

\section{Expression analysis of CACNA1I in human tissues}

To explore the expression of CACNA1I in human tissues, we downloaded the RNA-seq-based expression data from the study of Fagerberg et al. [29] Briefly, expression data from 27 different tissues of 95 human individuals were used.

\section{Results}

\section{A SNP in CACNA1I is significantly associated with SCZ in Chinese population}

We successfully genotyped the three selected SNPs (rs4748478, rs1006737 and rs4522708) in most of our samples. The overall genotyping call rate of these three SNPs exceeded 0.99. Hardy-Weinberg equilibrium analysis showed that all of the three SNPs are in Hardy-Weinberg equilibrium. Single SNP association test indicated that rs4748478 and rs1006737 were not associated with SCZ in our sample $(P>0.05)$. However, we found that rs4522708 is significantly associated with SCZ (reference allele: A, $\mathrm{OR}_{\mathrm{A}}$ allele $=1.19, P=0.0145$ ) (Table 1 ). Considering that we tested three SNPs in this study, we corrected $P$ values using Bonferroni correction. Again, we found rs4522708 still showed significant association with SCZ even Bonferroni correction was applied $(P=0.0435)$, suggesting this SNP is also associated with SCZ in Chinese population.

SNP rs4522708 has two alleles, A and G. The risk allele of rs4522708 in our sample is A allele $\left(\mathrm{OR}_{\mathrm{A}}\right.$ allele $=1.19$, $P=0.0145)$. Of note, we found the risk allele of rs4522708 is also the A allele in PGC2 $\left(\mathrm{OR}_{\mathrm{A}}\right.$ allele $=1.071$, $\left.P=2.41 \times 10^{-10}\right)(35,476$ schizophrenia cases and 46,839 controls). We thus performed a meta-analysis through combing our samples (1615 cases and 1597 controls) with samples from PGC2 (35,476 schizophrenia cases and 46,839 controls). A total of 37,091 SCZ cases and 48,436 controls were included in the meta-analysis. Heterogeneity test showed there was no heterogeneity in the combined samples $(P>0.05)$. The meta-analysis (fixed-effect model) further strengthened the association between rs4522708 and SCZ $\left(\mathrm{OR}_{\mathrm{A}}\right.$ allele $=1.074$, two-tailed $\left.P=6.26 \times 10^{-11}\right)$. Taken together, these results suggest that rs4522708 may represent an authentic risk variant for SCZ.

\section{CACNA1I was significantly up-regulated in SCZ cases compared with controls}

Our genetic association results indicate that CACNA1I is also associated with $\mathrm{SCZ}$ in Chinese population. To further explore the potential role of $C A C N A 1 I$ in schizophrenia pathogenesis, we examined $C A C N A 1 I$ expression in hippocampus of SCZ cases and controls. We found that CACNA1I was significantly up-regulated in hippocampus of SCZ cases compared with controls $(P=0.019$, Fig. 1$)$, suggesting dysregulation of CACNA1I in schizophrenia cases. This expression analysis provided further evidence that supports the involvement of CACNAII in schizophrenia.

\section{CACNA1/ was highly expressed in human brains compared} with other tissues

We found that CACNA1I was highly expressed in human brain tissues compared with other tissues (Fig. 2). In fact, $C A C N A 1 I$ has the highest expression level in human brains, suggesting this gene may have a role in human brain.

\section{Discussion}

Recent large-scale GWAS of schizophrenia (PGC2) have frequently reported that calcium signaling pathway genes were associated with schizophrenia. Of note, genetic variants in CACNA1C and CACNA1I reached genomewide significance level in PGC2. In addition, previous study also showed that $C A C N B 2$ gene was a risk gene for $\mathrm{SCZ}$. Though the associations between these three calcium signaling pathway genes and SCZ have been

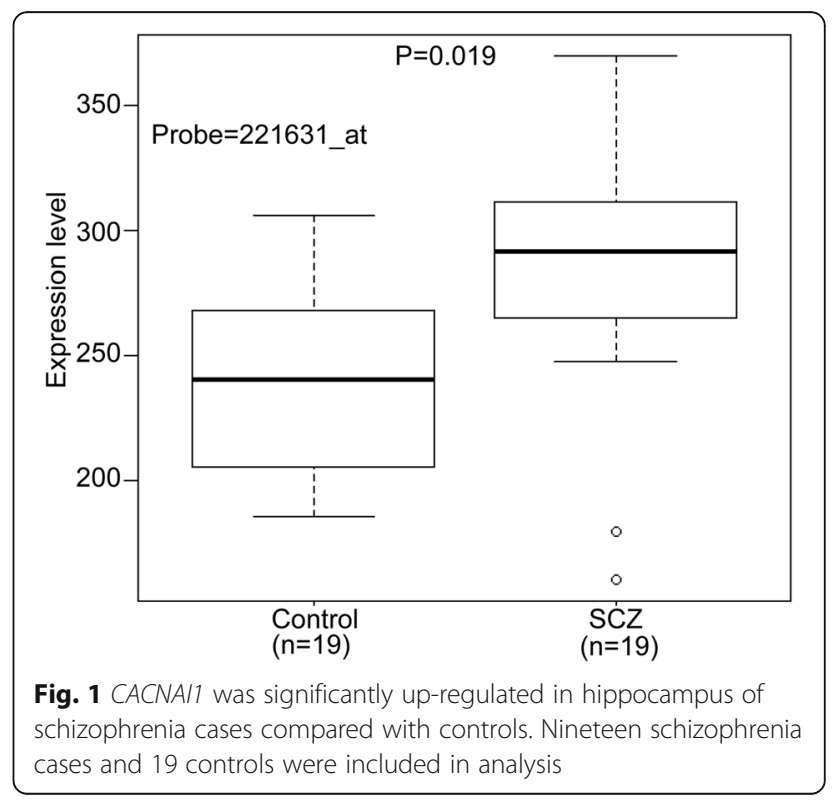




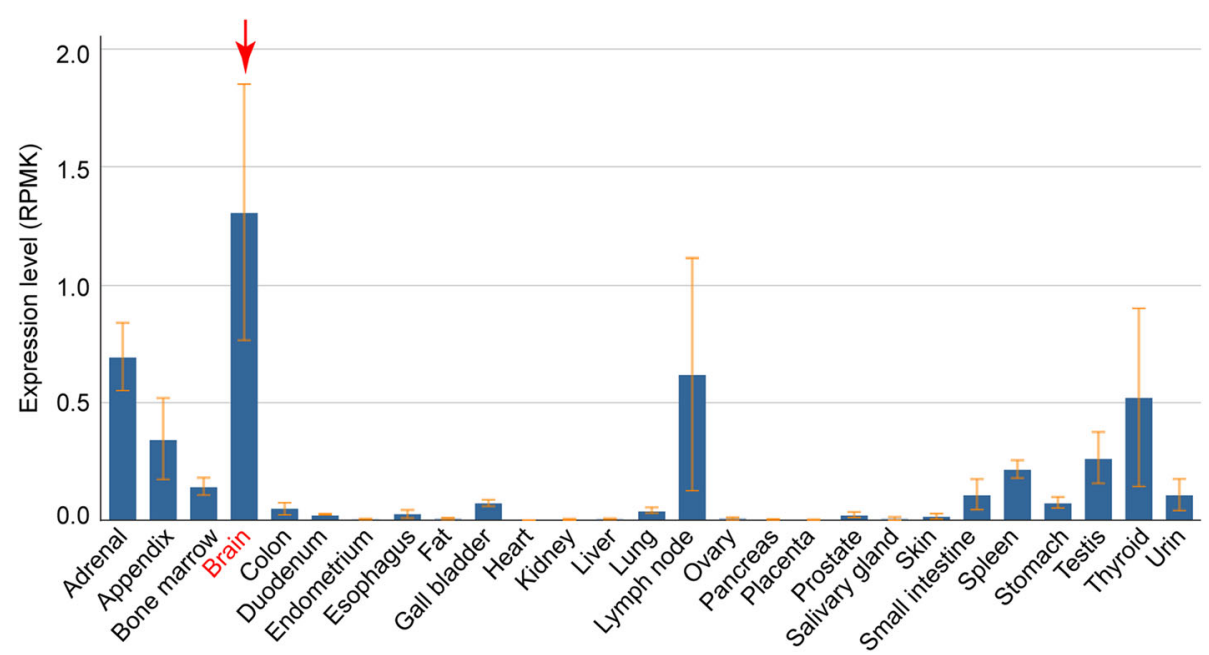

Fig. 2 CACNA/1 is highly expressed in human brain tissues compared with other tissues. The arrowhead shows the human brain tissue

frequently reported, we noticed that most of the studies were from populations of European ancestry. It is not known if these three calcium signaling pathway genes were also associated with SCZ in Han Chinese. In this study, we genotyped three SNPs in $C A C N B 2, C A C N A 1 C$ and CACNA1I in $1615 \mathrm{SCZ}$ cases and 1597 controls (All of the subjects were Han Chinese). We found that a SNP in CACNA1I is significantly associated with SCZ in our Chinese sample, suggesting CACNA1I may also represent a risk gene for $\mathrm{SCZ}$ in Chinese population. To further explore the potential role of CACNA1I in SCZ, we examined expression analysis and found that expression of CACNA1I was significantly up-regulated in SCZ cases compared with controls, implying dysregulation of $C A C$ NA1I may have a role in SCZ.

Of note, a recent study also showed that genetic variants in CANCA1I were significantly associated with SCZ in Uighur Chinese population [30]. Six SNPs (rs132575, rs713860, rs738168, rs136805, rs5757760 and rs5750871) were found to be associated with SCZ in the study of $\mathrm{Xu}$ et al. [30] We studied the linkage disequilibrium between these six SNPs and the SNP investigated in our study (i.e., rs4522708). We found that rs4522708 is linked with two previous reported SNPs (rs713860 and rs738168) (Fig. 3). However, rs4522708 is not linked with other significant SNPs reported in previous study [30]. These LD analysis results suggested that several independent genetic variants in CACNAI1 were associated with SCZ. The observation of significant association between CACNA1I and SCZ in two independent Chinese samples implies that CACNAI1 may represent an authentic risk gene for SCZ. Consistent with these findings, a recent trans-ancestry meta-analyses of Chinese and PGC2 samples (a total of 43,175 cases and 65,166 controls) provided further evidence for the association between CACNB2, CACNA1C, CACNA1I genes and schizophrenia [15]. These results strongly suggest that neuronal calcium signaling pathway plays a pivotal role in schizophrenia.

There were several limitations of this study. First, only one SNP was selected for each of the three calcium signaling pathway genes in this study. Thus, the SNP coverage is limited. We could not exclude if other SNPs in CACNB2 and CACNA1C were associated with SCZ.

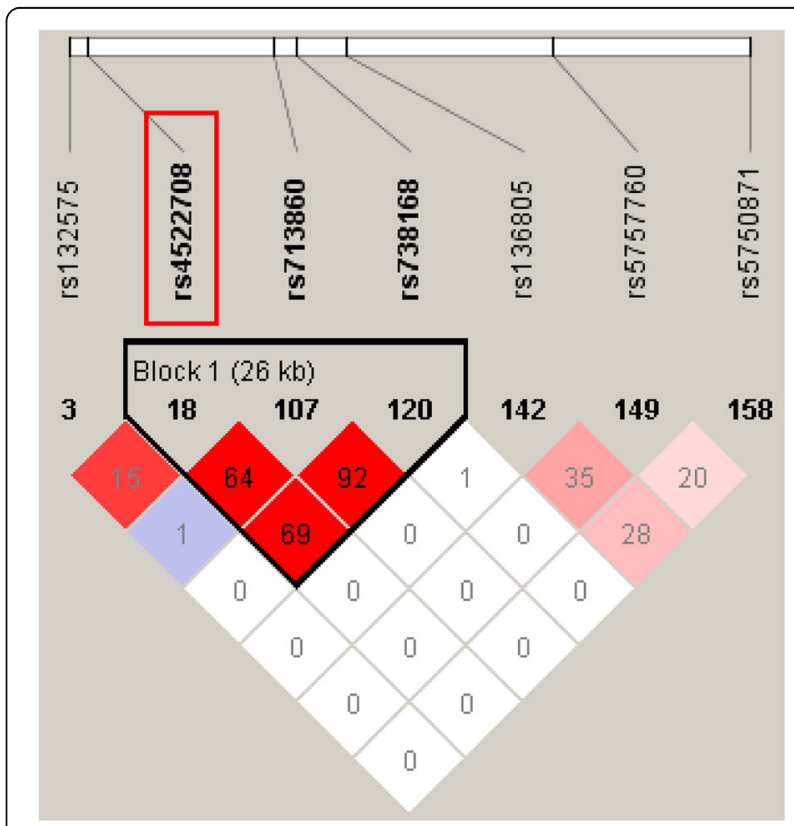

Fig. 3 Linkage disequilibrium analysis among the SNP investigated in this study (rs4522708) and the SNPs that were significantly associated with schizophrenia in previous study. The significant SNP observed in this study (rs4522708) is marked with red box. The values of linkage disequilibrium ( $r$ [2]) were shown in the box 
Second, the sample size is relatively small in this study. Further replication study with larger sample size is needed to validate our results. Taken together, our study showed that CACNA1I is also associated with SCZ in Chinese population. Our results provide further evidence that support the association of CACNA1I with SCZ.

\section{Acknowledgements}

We thank all of the participants in the study. This work was supported by the Key Research Project of Yunnan Province (2017FA008 to X.J.L).

\section{Authors' contributions}

$X J L$ conceived and designed the study. YJX, DH and LW performed the genotyping and analyzed the data. XJL wrote the paper. All authors reviewed the manuscript. All authors read and approved the final manuscript.

\section{Competing of interest}

The authors declare no competing financial interests.

\section{Publisher's Note}

Springer Nature remains neutral with regard to jurisdictional claims in published maps and institutional affiliations.

\section{Author details \\ 'Clinical Laboratory, Sichuan Academy of medical sciences \& Sichuan provincial people's hospital, Chengdu 610072, China. ${ }^{2}$ Key Laboratory of Animal Models and Human Disease Mechanisms of the Chinese Academy of Sciences \& Yunnan Province, Kunming Institute of Zoology, Chinese Academy of Sciences, Kunming, Yunnan 650223, China. ${ }^{3}$ Clinical Laboratory, The fourth people's hospital of Chengdu, Province, Chengdu, Sichuan 610000, China.}

Received: 12 November 2017 Accepted: 17 December 2017 Published online: 02 January 2018

\section{References}

1. Saha S, Chant D, Welham J, McGrath J. A systematic review of the prevalence of schizophrenia. PLoS Med. 2005;2:e141.

2. Andreasen NC. Symptoms, signs, and diagnosis of schizophrenia. Lancet. 1995:346:477-81.

3. Owen MJ, Sawa A, Mortensen PB. Schizophrenia. Lancet. 2016;388:86-97.

4. Sullivan PF, Kendler KS, Neale MC. Schizophrenia as a complex trait: evidence from a meta-analysis of twin studies. Arch Gen Psychiatry. 2003;60:1187-92.

5. Sullivan PF, Daly MJ, O'Donovan M. Genetic architectures of psychiatric disorders: the emerging picture and its implications. Nat Rev Genet. 2012;13:537-51.

6. Lewis CM, Levinson DF, Wise LH, DeLisi LE, Straub RE, Hovatta I, et al. Genome scan meta-analysis of schizophrenia and bipolar disorder, part II: schizophrenia. Am J Hum Genet. 2003;73:34-48.

7. Ng MY, Levinson DF, Faraone SV, Suarez BK, DeLisi LE, Arinami T, et al. Meta-analysis of 32 genome-wide linkage studies of schizophrenia. Mol Psychiatry. 2009;14:774-85

8. Allen NC, Bagade S, McQueen MB, loannidis JP, Kavvoura FK, Khoury MJ, et al. Systematic meta-analyses and field synopsis of genetic association studies in schizophrenia: the SzGene database. Nat Genet. 2008:40:827-34.

9. O'Donovan MC, Craddock N, Norton N, Williams H, Peirce T, Moskvina V, et al. Identification of loci associated with schizophrenia by genome-wide association and follow-up. Nat Genet. 2008;40:1053-5.

10. Yue WH, Wang HF, Sun LD, Tang FL, Liu ZH, Zhang HX, et al. Genome-wide association study identifies a susceptibility locus for schizophrenia in Han Chinese at 11p11.2. Nat Genet. 2011;43:1228-31

11. Shi Y, Li Z, Xu Q, Wang T, Li T, Shen J, et al. Common variants on 8p12 and 1q24.2 confer risk of schizophrenia. Nat Genet. 2011;43:1224-7.

12. Ripke S, O'Dushlaine C, Chambert K, Moran JL, Kahler AK, Akterin S, et al. Genome-wide association analysis identifies 13 new risk loci for schizophrenia. Nat Genet. 2013:45:1150-9.

13. Schizophrenia Working Group of the Psychiatric Genomics Consortium. Biological insights from 108 schizophrenia-associated genetic loci. Nature. 2014;511:421-7.
14. Juraeva D, Haenisch B, Zapatka M, Frank J, Witt SH, Muhleisen TW, et al. Integrated pathway-based approach identifies association between genomic regions at CTCF and CACNB2 and schizophrenia. PLoS Genet. 2014;10:e1004345.

15. Li Z, Chen J, Yu H, He L, Xu Y, Zhang D, et al. Genome-wide association analysis identifies 30 new susceptibility loci for schizophrenia. Nat Genet. 2017:49:1576-83.

16. Luo XJ, Diao HB, Wang JK, Zhang H, Zhao ZM, Su B. Association. Of haplotypes spanning PDZ-GEF2, LOC728637 and ACSL6 with schizophrenia in Han Chinese. J Med Genet. 2008:45:818-26.

17. Li M, Luo XJ, Xiao X, Shi L, Liu XY, Yin LD, et al. Allelic differences between Han Chinese and Europeans for functional variants in ZNF804A and their association with schizophrenia. Am J Psychiatry. 2011;168:1318-25.

18. Green EK, Grozeva D, Jones I, Jones L, Kirov G, Caesar S, et al. The bipolar disorder risk allele at CACNA1C also confers risk of recurrent major depression and of schizophrenia. Mol Psychiatry. 2010;15:1016-22.

19. Nyegaard M, Demontis D, Foldager L, Hedemand A, Flint TJ, Sorensen KM, et al. CACNA1C (rs1006737) is associated with schizophrenia. Mol Psychiatry. 2010;15:119-21.

20. He K, An Z, Wang Q, Li T, Li Z, Chen J, et al. CACNA1C, schizophrenia and major depressive disorder in the Han Chinese population. Br J Psychiatry. 2014;204:36-9.

21. Zheng F, Zhang Y, Xie W, Li W, Jin C, Mi W, et al. Further evidence for genetic association of CACNA1C and schizophrenia: new risk loci in a Han Chinese population and a meta-analysis. Schizophr Res. 2014:152:105-10.

22. Ivorra JL, Rivero O, Costas J, Iniesta R, Arrojo M, Ramos-Rios R, et al. Replication of previous genome-wide association studies of psychiatric diseases in a large schizophrenia case-control sample from Spain. Schizophr Res. 2014;159:107-13.

23. Purcell S, Neale B, Todd-Brown K, Thomas L, Ferreira MA, Bender D, et al. PLINK: a tool set for whole-genome association and population-based linkage analyses. Am J Hum Genet. 2007;81:559-75.

24. Viechtbauer W. Conducting Meta-Analyses in R with the metafor Package. J Statistical Software. 2010;36 https://doi.org/10.18637/jss.v18036.i18603.

25. Luo XJ, Li M, Huang L, Steinberg S, Mattheisen M, Liang G, et al. Convergent lines of evidence support CAMKK2 as a schizophrenia susceptibility gene. Mol Psychiatry. 2014;19:774-83.

26. Lanz TA, Joshi JJ, Reinhart V, Johnson K, Grantham LE, 2nd, Volfson D. STEP Levels are unchanged in pre-frontal cortex and associative striatum in postmortem human brain samples from subjects with schizophrenia, bipolar disorder and major depressive disorder. PLoS One 2015; 10: e0121744.

27. Abecasis GR, Altshuler D, Auton A, Brooks LD, Durbin RM, Gibbs RA, et al. A map of human genome variation from population-scale sequencing. Nature. 2010:467:1061-73.

28. Barrett JC, Fry B, Maller J, Daly MJ. Haploview: analysis and visualization of LD and haplotype maps. Bioinformatics. 2005:21:263-5.

29. Fagerberg L, Hallstrom BM, Oksvold P, Kampf C, Djureinovic D, Odeberg J, et al. Analysis of the human tissue-specific expression by genome-wide integration of transcriptomics and antibody-based proteomics. Mol Cell Proteomics. 2014;13:397-406.

30. Xu W, Liu Y, Chen J, Guo Q, Liu K, Wen Z, et al. Genetic risk between the CACNA1I gene and schizophrenia in Chinese Uygur population. Hereditas. 2017;155:5.

\section{Submit your next manuscript to BioMed Central} and we will help you at every step:

- We accept pre-submission inquiries

- Our selector tool helps you to find the most relevant journal

- We provide round the clock customer support

- Convenient online submission

- Thorough peer review

- Inclusion in PubMed and all major indexing services

- Maximum visibility for your research

Submit your manuscript at www.biomedcentral.com/submit
Biomed Central 